\title{
Maternal Dendritic Cells Influence Fetal Allograft Response and Tolerance
}

Citra Mattar ( $\nabla$ citramattar@nus.edu.sg )

National University of Singapore

Karthikeyan Kandasamy

Department of Obstetrics and Gynaecology, National University of Singapore

Nuryanti Binti Johana

KK Women's and Children's Hospital

\section{Lay Geok Tan}

National University of Singapore

Yvonne Tan

KK Women's and Children's Hospital

Julie Yeo

KK Women's and Children's Hospital

\section{Zhihui Li}

Genome Institute of Singapore, Agency for Science, Technology and Research (A*STAR)

Jiayu Koh

Genome Institute of Singapore, Agency for Science, Technology and Research (A*STAR)

Florent Ginhoux

Singapore Immunology Network, Agency for Science, Technology and Research

https://orcid.org/0000-0002-2857-7755

Jerry Chan

KK Hospital

\section{Mahesh Choolani}

Department of Obstetrics \& Gynaecology, Yong Loo Lin School of Medicine, National University of Singapore

\section{Article}

Keywords:

Posted Date: January 25th, 2022

DOI: https://doi.org/10.21203/rs.3.rs-1183982/v1 
License: (c) (i) This work is licensed under a Creative Commons Attribution 4.0 International License. Read Full License 
Karthikeyan Kandasamy ${ }^{1}$, Nuryanti Binti Johana ${ }^{3}$, Lay Geok Tan ${ }^{1,2}$, Yvonne Tan ${ }^{3}$, Julie Yeo Su Li ${ }^{3}$, Zhihui Li ${ }^{5}$, Jiayu Koh ${ }^{5}$, Florent Ginhoux ${ }^{6,7,8}$, Jerry KY Chan ${ }^{1,3,4}$, Mahesh Choolani ${ }^{1,2}$, Citra NZ Mattar ${ }^{1,2,{ }^{*}}$

1. Experimental Fetal Medicine Group, Department of Obstetrics and Gynaecology, Yong Loo Lin School of Medicine, National University of Singapore, 119228 Singapore

2. Department of Obstetrics and Gynaecology, National University Hospital, National University Health System, Singapore

3. Reproductive Medicine, KK Women's and Children's Hospital, Singapore

4. Cancer and Stem Cell Biology Program, Duke-NUS Graduate Medical School, Singapore

5. Genome Research Informatics \& Data Science Platform, Genome Institute of Singapore, Agency for Science Technology and Research, Singapore

6. Singapore Immunology Network (SlgN), Agency for Science, Technology and Research (A*STAR), Singapore

7. Translational Immunology Institute, Singhealth/Duke-NUS Academic Medical Centre, the Academia, Singapore

8. Shanghai Institute of Immunology, Shanghai JiaoTong University School of Medicine, Shanghai, China

*Corresponding author: Dr Citra NZ Mattar, Associate Professor, Experimental Fetal Medicine Group, Department of Obstetrics and Gynaecology, Yong Loo Lin School of Medicine, National University of Singapore, 1E Kent Ridge Road Singapore 119228, Telephone: +65-67722672, Fax: +65 6779 4753, email: citramattar@nus.edu.sg

\section{Acknowledgments:}

This study was funded by the Singapore Ministry of Health National Medical Research Council NMRC/CSA INV/0012/2016. CM is supported by grants from the NMRC/TA/0003/2012, NMRC/CSA-INV/0012/2016, NMRC/CSAINV20nov-0016. JKYC is supported by grants from NMRC/CSA-SI-008-2016, Singapore, MC is supported by grants from NMRC/CSA/0059/2014, Singapore.

\section{Author Contributions:}

KK, LGT, CNZM designed and performed research, analyzed data, and wrote the manuscript; NBJ, YT, JYSL, LZ, KJ performed research, analyzed data and wrote the manuscript, FG, MC, JKYC assisted with data analysis and revised the manuscript. CNZM supervised experimental design and manuscript preparation.

\section{Competing Interests:}

The authors have declared that no competing interest exists. 
Extended data and Supplementary Information are available for this paper.

17

\section{Materials \& Correspondence:}

19 Correspondence and requests for materials should be addressed to Dr. Citra NZ Mattar.

20

21 Data Availability Statements:

22 The datasets generated and/or analysed during the current study are available from the corresponding author 23 on reasonable request. 
Intrauterine hematopoietic cell transplantation (IUT), a promising therapy for congenital haematological disease, is limited by subtherapeutic donor cell chimerism (DCC). Microchimerism of maternal immune cells (MMc) trafficked into fetal IUT recipients may directly influence immune-mediated donor cell clearance. We investigated if maternal dendritic cell (DC) suppression reduces recipient alloresponsiveness to donor cells, improving DCC. IUT was performed at E14 in pregnancies resulting from crossing CD11c.DTR(B6) females and Balb/c males, with semiallogenic Balb/c or C57BL/6, or fully allogenic $\mathrm{C} 3 \mathrm{H}$ donor cells, $24 \mathrm{~h}$ after administering diphtheria toxin to the dam, transiently suppressing maternal DC. This resulted in reduced MMc in recipient fetuses, greatest after Balb/c transplantation, also associated with the highest DCC, and lowest with $\mathrm{C} 3 \mathrm{H}$. Maternal-derived Tcell receptor (TCR) clonotypes were enriched in IUT recipients and displayed substantially reduced diversity. Recipient pups with reduced MMc increased expression of regulatory T-cell subtypes, reduced expression of proinflammatory cytokines, and demonstrated enhanced TCR clonotype diversity. DCC was primarily related to donor cell type and not influenced by MMc. Our data indicate that donor cell origin and MMc are distinct factors determining IUT effectiveness. MMc independently influences DCC and recipient tolerance to donor cells and may present novel therapeutic targets to improve transplantation outcomes. 
40 In utero hematopoietic stem cell transplantation (IUT) represents potential cure for congenital hematological

41 disorders with advantages over conventional postnatal transplantation, particularly avoidance of myeloablation, ${ }^{1}$

42 but immune barriers cause poor engraftment, hampering clinical application..$^{2-5}$ Conventional dendritic cells 43 ( $\mathrm{CDC}$ ) activate $\mathrm{CD} 8$ and $\mathrm{CD} 4 \mathrm{~T}$-cells via subtypes $\mathrm{CDC} 1$ and $\mathrm{CDC} 2$ respectively, ${ }^{6-8}$ mediating antigen-specific 44 tolerance by altering helper T-cell (Th) balance ${ }^{9,10}$ and cytokine expression. ${ }^{11-13}$ Active maternal-fetal trafficking occurs throughout pregnancy, ${ }^{14,15}$ increasing after invasive procedures like IUT. ${ }^{16-18}$ Fetal alloresponsiveness to 46 IUT and subsequent donor cell chimerism (DCC) may be influenced by trafficked maternal cDC. . $^{19,20}$ To understand how microchimeric maternal immune cells (MMc) in fetal IUT recipients affect DCC , we investigated semi-allogenic and allogenic DCC following transient depletion of maternal CDC subtypes in a murine IUT model. We observed lower MMc, expanded recipient regulatory T-cells, and subdued recipient alloresponsiveness to donor cells. DCC, unaffected by reduced MMc, was highest with semi-allogenic (paternal) IUT at $>2 \%$ for 12 weeks, and retained hematopoietic phenotype. T-cell (TCR) and B-cell receptor (BCR) repertoire diversity was contracted within recovered MMc and expanded in recipient cells with semi-allogenic IUT; clonotype diversity was variably influenced by maternal $\mathrm{CDC}$ depletion. Our results demonstrate that $\mathrm{DCC}$ depends on donor cell origin, not MMc, and maternal and recipient alloresponsiveness can be manipulated via maternal cDC depletion. Unique maternal and recipient TCR/BCR repertoires may serve as therapeutic targets to improve transplantation outcomes. This clinically relevant data, similar to our previous study, ${ }^{16}$ supports paternal IUT for clinical trials and encourages short-term maternal immune suppression at IUT. 
Results

Maternal CD11C+MHC-II+DC depletion influenced trafficked maternal cells following IUT.

Diphtheria toxin (DT) administration to non-pregnant CD11c-DTR females depleted CD11c+MHC-II+cDC in spleen, bone marrow (BM), peripheral blood (PB) and uterus (Extended data Figure S1a). ${ }^{21}$ Baseline cDC (0.23-0.38\%) reached troughs (0.01-0.06\%) 2-7 days post-DT, returning to baseline on day 7 in BM and uterus (Figure 1a). cDC1 was $<0.1 \%$ at all timepoints, while $\mathrm{cDC} 2$ was returned to $0.86 \%$ in $\mathrm{BM}$ by day 7 . Other immune cells remained unaffected. Our IUT model (CD11c-DTR females crossed with BALB/c males) allowed us to track donor, maternal and recipient cells separately. Intrahepatic IUT utilising maternal (B6, mIUT), paternal (Balb/c, plUT) or allogenic C3H (alUT) cells produced survival rates in DT- and DT+ pups respectively of $92.0 \%$ and $76.9 \%$ (uninjected controls, $n=61$ ), $75.0 \%$ and $79.6 \%$ (plUT, $n=60$ ), $81.1 \%$ and $45.0 \%$ (mIUT, $n=31$ ), $100.0 \%$ and $46.7 \%$ (alUT, n=22, Figure 1b).

Next, we determined that microchimeric maternal cells (MMc) following DT+plUT and DT-pIUT were similar in BM (0.32-0.48\%) and PB (0.43-0.78\%), higher after DT+mIUT in BM than PB (4-8w, $p<0.05$, Figure 1c), and higher in DT-alUT than DT-pIUT (8w, p<0.05, Figure 1c,d). cDC was observed only in DT-IUT BM (0.03-0.08\%), and in DT+alUT and DT-alUT PB (0.04-0.08\%); cDC1 and cDC2 were detected only in DT-mIUT and DT-alUT BM (0.03-0.04\%, Figure 1d). Compared to DT-, we observed non-significant reductions in CD3, CD4, CD8, CD19 in DT+plUT and DT+mIUT BM, and higher CD3, CD4, CD8 in DT+alUT BM (Figure 1d), with nonsignificant increases in CD3, CD4, CD8 in DT+plUT and DT+mIUT PB, and reductions in all cells in DT+alUT, particularly NK1.1 $(10.4 \pm 10.4 \% \vee 29.9 \pm 10.7 \%, p<0.0001)$. Thus, maternal DC depletion influences MMc differently depending on IUT.

\section{Donor cell chimerism was highest following pIUT and hematopoietic markers were retained}

We observed higher donor cell chimerism (DCC) in BM (DCC ${ }^{\mathrm{BM}}$ ) in DT-pIUT than in DT-mIUT and DT-alUT recipients (0w, $\mathrm{p}<0.05)$. and higher $D C C$ in PB $\left(D C C^{P B}\right)$ in DT-pIUT compared to DT-alUT $(4-12 w, p<0.05)$. DTmIUT showed higher DCC ${ }^{\mathrm{PB}}$ than DT-alUT and DT+mIUT (0w, $\left.\mathrm{p}<0.05\right)$. With DT+plUT, DCC ${ }^{\mathrm{BM}}$ was maintained $>2 \%$ until $12 w$, though $D C C^{P B}$ declined to $<1 \%$ by $8 w\left(D C C^{B M} \vee D C C^{P B}\right.$ at $12 w, p<0.001$, Figure 1c). $D C C^{B M}$ in DT-mIUT and DT+mIUT remained $<1 \%$ while $\mathrm{DCC}^{\mathrm{PB}}$ declined rapidly from $11.07 \pm 0.07 \%$ (0w, DT-mIUT), and remained $<1 \%(0-12 w, D T+m I U T)$. DCC ${ }^{B M}$ and $D C C^{P B}$ in DT-alUT and DT+alUT remained $<1 \%(0-12 w)$. Recovered DCC ${ }^{\mathrm{BM}}$ and $\mathrm{DCC} \mathrm{PB}^{\mathrm{B}}$ showed similar proportions of Lin-Sca1+C-Kit+ (LSK), CD48+ and CD150+ indicating the presence of long-term repopulating HSC (Extended data Figure S1b). We deduced that postIUT maternal cell trafficking was an active process, as we observed significant reductions in CD3 and CD19 in recipient BM and PB compared to uninjected dams (all differences, $p<0.0001$ ), except for NK1.1 which was 
higher in alUT recipients than controls $(p<0.0001$, Extended data Figure S2a). Thus, DCC appears to be independent of MMc.

\section{Maternal DC depletion increased recipient Treg and reduced cytokine expression associated with} alloreactivity.

We next examined the impact of maternal DC depletion on DCC and alloresponsiveness of IUT recipients. In DT+plUT, CD19 was significantly higher in $\operatorname{DCC}^{\mathrm{BM}}(4-8 \mathrm{w}, \mathrm{p}<0.05)$ and in $\mathrm{DCC}^{\mathrm{PB}}(0-12 \mathrm{w}, \mathrm{p}<0.05)$ than other cells. In DT-DCC ${ }^{\mathrm{PB}}, \mathrm{CD} 19$ was more prevalent at $0 \mathrm{w}(\mathrm{p}<0.0001$, Figure 2a, b). Within corresponding MMc, maternal CD3 was significantly lower in DT+pIUT compared to DT-pIUT (where it is the most prevalent cell) at $0-12 w(p<0.005)$ and was also higher than cDC subsets in DT+pIUT $(4 w, p<0.05)$; no other differences were observed in MMc between groups (Figure 2c, d). DT+plUT and DT-pIUT produced higher CD19 than other immune cells in BM and PB ( $p<0.005$ at all timepoints, Figure 2e,f) with no differences between groups.

When examined for tolerance, re-exposure of DT+pIUT splenocytes to Balb/c cells elicited greater expression of CD4 effector memory (Tm), regulatory (Treg) and CD25+Treg compared to DT-plUT and untreated controls $(p<0.001$, Figure $\mathbf{2 g}$ ), and re-exposure to B6 cells produced higher CD4 Treg compared to DT-pIUT and controls $(p<0.001)$. DT+plUT recipients showed greater fold-change in CD8 central Tm, effector (Teff), Treg and CD62L+CD25+Treg $(p<0.001)$ when challenged with B6 cells, compared with controls and DT- recipients (Figure 2h). DT-plUT produced greater fold-changes in cytokines associated with helper T-cells, ${ }^{22}$ when stimulated with Balb/c or B6 cells, while a relatively lower response was observed for DT+plUT (Figure 2i-n). Postnatal boost of maternal cells increased CD3 and decreased CD19 levels only in lymph nodes in DT+pIUT (Extended data Figure S2b-f), but no donor-specific antibodies were observed following IUT or postnatal boost (Extended data Figure S2g).

Though $\mathrm{DCC} \mathrm{C}^{\mathrm{BM}}$ and $\mathrm{DCC} \mathrm{C}^{\mathrm{PB}}$ were $<0.2 \%$ in $\mathrm{DT}+$ mIUT, we were able to analyse donor immune cell components. $\mathrm{DCC}^{\mathrm{BM}}$ in DT+mIUT contained higher CD3, CD8, CD19 than $\mathrm{CDC}, \mathrm{NK} 1.1(\mathrm{p}<0.05)$, and compared to DT-mIUT, $D C^{B M}$ contained higher $C D 3$, while $D C C^{P B}$ showed lower $C D 3, C D 8(p<0.05$, Figure $3 \mathbf{a}, \mathbf{b})$. There were no differences in MMc (Figure 3c,d) and recipient immune profile (Figure 3e,f) in DT+mIUT BM or PB compared to DT-mIUT.

DT+mIUT elevated CD4 effector Tm, CD4 and CD8 Teff, and CD4 and CD8 CD25+Treg, CD62L+CD25+Treg when stimulated with Balb/c cells only (Figure $\mathbf{3 g}, \mathbf{h}$ ); only low-level responses to B6 cells were elicited. Cytokine expression was much lower in mIUT than plUT recipients ( $<6$-fold increase over untreated controls). DT+mIUT produced a higher expression of largely inhibitory cytokines (IL5, IL6, IL10, FOXP3, TGFß2) in combinations associated with Th2, Th1/Th2, Th17 cells, following B6 and Balb/c stimulation (Figure 3i-n). 
In contrast, DT+alUT and DT-alUT resulted in only microchimeric $\mathrm{DCC} \mathrm{BM}^{\mathrm{BM}}$ and $\mathrm{DCC} \mathrm{CB}^{\mathrm{PB}}$, with similar donor immune cell profiles. No differences in MMc, trafficked maternal immune cell profile or recipient immune cell profile were observed (Extended data Figure S3a-f). Significant fold-change increases in CD4 central and effector Tm, and CD4 and CD8 Teff, CD25+Treg and CD62L+CD25+Treg were observed in DT+alUT (Extended data Figure S3g,h), which also significantly increased expression of all cytokines except FOXP3 when stimulated with $\mathrm{C} 3 \mathrm{H}$, compared to DT-alUT, implying proimmune helper T cell enhancement. alUT produced cytokine responses close to control levels when stimulated with B6 and Balb/c, except for IL-17a, IL-17f, IL-22, augmented with DT+alUT (Extended data Figure S3i-n).

We attempted to evaluate regulatory B-cells (Breg) which lack unique phenotypic markers. ${ }^{23,24}$ pIUT and mIUT augmented IL10, IL5, IL6, TGF-32, FOXP3 expression (Figure 2j,m; Figure 3j,m) suggesting the presence of activated Breg in response to IUT. There were no differences in CD19 post-transplantation (Figure 2e,f; Figure 3e,f). Thus, maternal DC depletion may promote a tolerogenic response through both Treg and Breg.

\section{IUT and DC depletion influence maternal and recipient T- and B-cell receptor repertoire diversity}

To further parse maternal and recipient immune interactions, we analysed gene expression profile and T-cell (TCR) and B-cell (BCR) receptor repertoires in MNC isolated from pIUT and mIUT recipients. Top 3000 up- and down-regulated genes in each group - uninjected (DT-, n=17), DT-mIUT ( $n=7)$, DT-pIUT ( $n=5)$, DT+plUT $(n=5)$ - were identified by enrichment score $(E S)>1.3$. Within treatment groups, recipient and maternal cells shared 483-736 common genes (Figure 4a). With mIUT, 26/174 enriched recipient gene clusters and 25/171 maternal clusters represented RNA and protein metabolism, hemopoiesis and immune system development, also represented by $21 / 169$ recipient clusters and 49/177 maternal clusters following pIUT. With DT+plUT, 20/153 recipient clusters additionally represented T-cell regulation. Upregulated DT-pIUT and DT+plUT Maternal clusters represented mitogen-activated protein kinases (MAPK) cascades, T-cell activation, and immune system development. All groups shared 48 common genes, of which 6/40 highly enriched clusters represented cytokine stimulus response, immune system regulation, B-cell mediated immunity and adaptive immune response (Figure 4b).

Compared to uninjected controls, retrieval frequency of maternal-derived TCR and BCR clonotypes increased from $0.02 \%$ to $2.04-3.13 \%$ following mIUT and pIUT respectively (Table 1), and these showed reduced diversity, with Hill numbers (order 1, exponential of Shannon-Wiener indices) $)^{25}$ of $18-29$, compared to 941 in controls. DT+pIUT decreased maternal clonotype retrieval by 17.3 -fold and increased diversity towards baseline (Hill number of 447). In contrast, mIUT and pIUT recipient-derived clonotypes (retrieval frequency $0.02-0.15 \%$ ) 
showed increased diversity (Hill numbers 528-3728 v 64 in controls). DT+plUT reduced diversity in recipients (Hill numbers from 3728 to 1637).

Maternal- and recipient-derived TCR and BCR clonotypes were similar in uninjected pups; BCR immunoglobulin heavy (IGH), к (IGK), $\lambda$ (IGL) chains made up $~ 81 \%$ of maternal and $75 \%$ of recipient clonotypes (Figure 4c). Expansion of TCR $\beta$-chain (TRB) clonotypes and reduction in IGH were observed among maternal-derived clonotypes following mIUT and DT-plUT. DT+plUT produced further increases in TRB, TRA ( $\alpha$-chain), TRD ( $\delta$ chain), IGH, IGK relative to DT-pIUT. Among recipient-derived clonotypes, we observed expanded TRB, TRG ( $\mathrm{\gamma}$-chain), and reduced IGH, IGK following mIUT and pIUT, and DT+pIUT further reduced TRB and increased IGK and IGH.

Mostly higher-order (3+) clonotypes were encountered with IUT (Figure 4d,e). Top 20\% of clonotypes (Quantile 1, Q1) were most abundant in uninjected controls, and clonotypes in Q2 to Q5 were more abundant in IUT recipients. Individual abundances of top 5 maternal-derived clonotypes in each group were expanded with IUT and diminished with DT+ (Figure 4d), while recipient-derived top 5 clonotypes were most abundant in uninjected pups (Figure 4e). We observed a large number of public maternal-derived clonotypes between DT+plUT and controls (Figure 4f), and a substantial number of public recipient-derived clonotypes between mIUT, pIUT and DT+plUT (Figure 4g).

Complementarity-determining region 3 (CDR3) incorporates the VDJ recombination junctions, accounting for most of the repertoire variation mediating specific antigen recognition. ${ }^{26}$ CDR3 regions of naïve and antigenexperienced clonotypes are longer and shorter respectively, reflecting antigen-driven selection. ${ }^{27}$ We found a significant shift towards longer CDR3 in maternal TRG (from mIUT) and TRA (DT-pIUT, DT+plUT), and shorter CDR3 in maternal TRB (DT+pIUT), recipient TRA (mIUT), and maternal and recipient IGH (all groups). Analyses of gene segment usage to functionally characterize these clonotypes by hierarchical clustering indicate similarities in V/J-segment usage between pIUT, DT+plUT and mIUT recipient-derived clonotypes and DT+pIUT maternal-derived clonotypes, where IGH, IGK, TRA, TRB V/J-segments, and TRG and TRD J-segments, were upregulated and TRA J-segments were downregulated. We also observed uniqueness in $\mathrm{V} / \mathrm{J}$-segment usage between uninjected control, mIUT and pIUT clonotypes (Extended data Figure S5a). Functionality of the top 20 shared clonotypes, assessed by comparing CDR3 amino acid sequences to protein databases using Tomtom, ${ }^{28}$ revealed motif enrichment corresponding to production of Ras GTPase activating proteins, MAPK substrates, and signal transduction molecules, among others (Extended data Figure S5b). These data show that both maternal and recipient immune cells respond to donor cells and that only maternal TCR and BCR diversity is affected by maternal DC depletion. 


\section{Discussion}

To our knowledge, this is the first report of maternal immune cells "primed" on exposure to donor cells influencing recipient immune responses to IUT. We demonstrated that DT+plUT reduced trafficking of maternal CDC, expanded regulatory T-cells and restricted pro-immune cytokines, contrasting with largely alloreactive responses to mIUT and alUT. Inducing the appropriate balance of Th1/Th17/Th2, which influence allograft tolerance or rejection, ${ }^{22,29}$ may be critical to improving IUT transplantation tolerance. Maternal TCR/BCR clonotypes displayed substantially reduced diversity post-IUT, restored with DC depletion, while recipient-derived TCR enrichment was unaffected. Our findings indicate that DCC and MMc are separate yet equally important determinants of IUT effectiveness. pIUT produced the highest and most persistent DCC. In contrast to other reported models using maternal donor cells, ${ }^{30,31}$ the model for IUT clinical trial NCT02986698, we observed microchimerism post-mIUT despite reduced MMc, similar to our earlier work. ${ }^{16}$ Thus, DCC appears primarily dependent on cell origin, not MMc. We demonstrated that maternal cell trafficking is an active process, the quality of which appears to influence the fetal recipient's immune response to donor cells. Taken together, pIUT resulted in both highest DCC and lowest MMc, and maternal DC depletion further dampened recipient alloresponsiveness through upregulated Treg, the possible presence of Breg, and downregulated proimmune Th cells. alUT produced both poorest DCC and most robust alloresponsiveness, contrasting with data from other IUT models in which allogenic donor cells have a competitive advantage. ${ }^{32-36}$ Although reduced MMc was associated with increased Treg and Breg cytokine expression, upregulated Teff and an overwhelmingly proimmune cytokine response were present, expediting allogeneic cell rejection. Maternal DC may also present donor antigen to recipient immune cells, influencing the quality of immune response. Enhanced Treg production following IUT, particularly of CD62L+CD25+FOXP3+ Tregs, protects against graft-v-host disease, ${ }^{37,38}$ and together with putative Breg (expressing IL10, IL5, IL6, FOXP3, TGF- $\beta$ ), probably influenced the resulting tolerogenic or immunogenic responses. ${ }^{23,24}$ Human transplantation data indicate that persistent recipient cells inhibit donor haematopoiesis and TCR reconstitution. ${ }^{39-41}$ IUT adds an interesting dimension to this, wherein the maternal immune system is the other "recipient" interacting with donor cells, and both maternal and recipient immune systems may impact long-term engraftment and maintenance of tolerance. 
Trafficked maternal TCR and BCR clonotypes showing reduced diversity, variable CDR3 lengths and V- and Jsegment usage indicate dynamic acquisition of allo-specificity, further underscoring the "sensitization" of maternal cells to transplanted donor cells. Donor cells transplanted directly into the fetus may leak into maternal circulation and participate in maternal immune modelling, selecting and expanding particular clonotypes for trafficking, similar to native fetal microchimerism. ${ }^{42}$ The return of DT+ maternal clonotypes to higher baseline diversity supports this postulation. Increased recipient TCR diversity confirm that treated fetuses mount antigenic responses even to haploidentical cells. Clinically-poor responders to postnatal HSCT show lower TCR diversity, ${ }^{26,43,44}$ thus recipient TCR repertoire may be a useful biomarker of transplant rejection..$^{45}$

Our murine model permitted specific depletion of antigen-presenting CDC in pregnant mice, for which there are no published data. CDC depletion at E13 would have endured for the remainder of the pregnancy (parturition E20), resulting in naïve maternal CDC trafficked to fetal recipients. Of particular interest is depletion of uterine DC, a unique subtype critical to pregnancy-related tolerance and rejection, ${ }^{46}$ capable of eliminating fetal cells, which concentrate in maternal circulation towards parturition, ${ }^{42}$ while not rejecting the fetus. ${ }^{47,48}$ Allogenic fetal cells presented by uterine DC can prime maternal tolerance towards paternal antigens via expansion or elimination of alloreactive or regulatory lymphocytes. ${ }^{49-51}$ Donor cells transplanted in midgestation likely leak into maternal circulation, prompting reciprocal trafficking of primed maternal cells. Indeed, with DC depletion, maternal TCR clonotypes showed markedly greater diversity, and pIUT and mIUT recipients expanded Treg subsets and upregulated less immunogenic cytokines. Further investigation into uterine DC may intriguingly reveal a unique target for in-vivo or ex-vivo modulation to enhance transplantation tolerance. $^{12}$

Our data raise the exciting possibility of utilising unique maternal and recipient TCR/BCR repertoires in multiple fashion for IUT, and by extension other cellular fetal therapies, by serving as biomarkers of engraftment and immune tolerance, as therapeutic targets to improve transplantation tolerance, e.g., by transducing regulatory sequences into high avidity clonotypes, and as prognosticators of transplantation outcomes. Robust graft 
tolerance required to maintain life-long engraftment carries several layers, ${ }^{52}$ that, in combination, may even permit repeat transplantation, including limiting maturation of high-avidity alloreactive T-cells and expanding high-avidity Treg. ${ }^{53-55}$ Selective expansion of regulatory TCR clonotypes with distinctly tolerogenic phenotypes 241 may be an individualizable strategy through which to achieve such tolerance. ${ }^{56}$ Bregs maintain immune 242 tolerance, but are more difficult to identify due to the absence of unique markers. ${ }^{57,58}$ Though maternal and 243 recipient TCR clonotypes underwent the greatest expansion, we observed an increase in recipient-derived BCR 244 clonotypes along with upregulated Breg-associated cytokines. This suggests that putative regulatory BCR 245 clonotypes are represented and may be valuable components of IUT tolerance. Our data may influence clinical 246 management even in the short-term, supporting the transplantation of paternal donor cells in clinical trials or 247 short-term maternal immune-suppression at the time of IUT, similar to immunotherapy for recurrent 248 miscarriages. ${ }^{59}$ Our work is limited by transplantation of a small donor inoculum and limited monitoring for 12 249 weeks, while pandemic restrictions curtailed our assessment of DT+mIUT and alUT immune repertoires. 250 Additionally, in the limited number of mIUT and alUT recipients harvested, donor chimerism was very low 251 which may affect accuracy of donor cell analyses. Transplantation of larger doses to achieve therapeutic DCC 252 and a longer surveillance of maternal/recipient immune profiles in larger animals will be informative for future 253 clinical translation. 
255

256

257

258

259

260

261

262

263

264

265

266

267

268

269

270

271

272

273

274

275

276

277

278

279

280

281

282

Reagents and antibodies. Roswell Park Memorial Institute Medium (RPMI 1640), fetal bovine serum (FBS), non-essential amino acids, GlutaMAX, 2-Mercaptoethanol, penicillin-streptomycin antibiotics and collagenase type IV powder were obtained from Gibco (Thermofisher Scientific, Waltham, MA). Mitomycin C was obtained from ALPS Pte Ltd (National University Hospital Pharmacy, Singapore). Diphtheria Toxin (DT) and bovine serum albumin (BSA) were obtained from Sigma-Aldrich (St. Louis, MO). Ficoll-Paque PLUS were obtained from GE Healthcare (Chicago, IL). RBC lysis buffer was obtained from Biolegend (San Diego, CA). Cytofix/Cytoperm solution, Perm/Wash buffer, staining buffer, brilliant stain buffer and fixable viability stain 620 were obtained from BD Biosciences (San Jose, CA). AutoMACS rinse solution was obtained from Miltenyi Biotec (Singapore). FastStart Universal SYBR green master mix (Roche Life Science, Germany) were obtained from Sigma-Aldrich. DNA oligos for qPCR were obtained from Integrated DNA Technologies (IDT, Singapore). VersaComp antibody capture compensation beads were obtained from Beckman Coulter (Singapore). Fluorescent-activated cell sorting (FACS) antibodies were obtained from BD Biosciences, Biolegend and Life Technologies (Thermofisher Scientific). A list of abbreviations used in the manuscript can be found in extended data table (S1).

Animal experiments. Inbred strains CD45.2 BALB/c $\left(\mathrm{H}-2 \mathrm{~K}^{d}\right)$ and $\mathrm{C} 3 \mathrm{H} / \mathrm{HeNTac}\left(\mathrm{H}-2 \mathrm{~K}^{\mathrm{k}}\right.$ referred to as $\left.\mathrm{C} 3 \mathrm{H}\right)$ were obtained from In Vivos (Singapore). CD45.1 C57BL/6 mice and CD11C-DTR female mice (B6.FVB1700016L21RikTg(Itgax-DTR/EGFP)57Lan/J) were purchased from The Jackson Laboratory (Bar Habor, ME) and maintained in a specific pathogen-free facility at NUS. BALB/c males and CD11c-DTR females were time-mated for IUT at E14. When performing animal experiments, pregnant mice were randomly chosen for IUT and for downstream experiments, as the probability of getting pregnant dams in a group of breeding mice occur by chance. All mouse experiments were performed according to IACUC (Institutional Animal Care and Use Committee) approved protocols (BR16-1203 and R16-1200) at the National University of Singapore (NUS), Singapore.

Transient DC suppression. CD11C-DTR females were given intraperitoneal injection of DT (5ng/g of body weight) and controls were given saline. The females were harvested on day $0,1,2,4$ and 7 days post injection, BM, PB, spleen and uterine horn organs were analysed for CD3, CD4, CD8, CD19, NK1.1, CD11C, MHC-II, XCR1 and CD172a immune parameters by flow cytometry. Conventional DC (cDC) were identified by the double positive population of MHC-II and CD11c. From CDC, CDC1(XCR1+) and CDC2 (CD172a+) cells were identified. 
Intrauterine transplantation (IUT) and postnatal boost. Fresh donor bone marrow mononuclear cells (BMMNC) were prepared as previously described. ${ }^{16}$ The day before IUT (E13), CD11c-DTR pregnant mothers (DT+) were given intraperitoneal DT injections (5ng/g body weight). DT- dams received of saline. IUT was performed on $\mathrm{E} 14$ and all fetuses received intrahepatic injection of $5 \mathrm{E}+6$ donor inoculum, as described previously. ${ }^{16}$ Maternal donor cell IUT (mIUT) was performed using B6 BM-MNC (CD45.1, H-2K ${ }^{b}$ ), BALB/c BM-MNC was administered in paternal donor cell IUT (pIUT) and C3H BM-MNC were used for complete allogenic donor cell IUT (alUT). Uninjected controls were offspring of DT+ or DT- dams which did not undergo intrauterine transplantation. After littering, pups were nursed by their mothers and weaned at 4 weeks. Fetal cells were harvested from $\mathrm{F} 1$ cross-bred hybrid pups sacrificed between postnatal weeks 1 to 12 to assess donor cell chimerism (DCC), trafficked maternal cell microchimerism (MMc) and fetal immunological responses in peripheral blood (PB) and BM. In selected offspring in each group, postnatal boost was performed with $5 \mathrm{E}+6$ donor cells after 12 postnatal weeks administered via retro-orbital route, without bone marrow ablation.

Isolation of MNC and FACS analysis. MNC were isolated from various organs and approximately $1 \mathrm{E}+6$ cells/tube were stained for surface markers to differentiate trafficked maternal cells (H2K-b+), and fetal recipient cells (double positive $\mathrm{H} 2 \mathrm{~K}-\mathrm{b}+, \mathrm{H} 2 \mathrm{~K}-\mathrm{d}+$ ) as reported. ${ }^{16}$ Donor cells were identified from fetal recipient cells by the expression of CD45.1+ (maternal donor cells), H2K-d+ (paternal donor cells), H2K-k+ (C3H donor cells) surface markers. Further, cells were stained for T cells (expressing CD3, CD4, CD8, NK1.1), B cells (CD19), dendritic cells (CD11c, MHC-II, XCR1, CD172a), HSC markers (Sca1, c-Kit, CD48, CD150), hematopoietic lineage markers (CD11b, GR1, TER119, CD3, CD19, B220). All antibody-stained cells were analyzed using BD X-20 Fortessa $^{\text {TM }}$ flow cytometer (BD Biosciences, Franklin Lakes NJ, accessed at the NUS Life Science Institute), The list of antibodies used for flow cytometry were provided in extended data table (S2). Each antibody was validated with positive control samples as per manufacturer's instructions. All raw data files were compensated and analysed using FlowJo ${ }^{\mathrm{TM}}$ software (FlowJo LLC, Ashland OR). Percentage of donor cells and maternal trafficked cells were calculated from the total live MNC. Percentage of immune populations were calculated from the respective total population of $\mathrm{MMc}$, donor cells and recipient cells and the percentage of HSC markers were calculated from the total donor cells.

Mixed lymphocyte reactivity assay (MLR) for functional T cell activity. Splenocytes (responder cells) were isolated from sacrificed F1 cross-bred IUT and naive (from uninterrupted pregnancies) pups. Lymphoid tissue MNC from BALB/c, B6 or $\mathrm{C} 3 \mathrm{H}$ mice, treated with mitomycin $\mathrm{C}(50 \mu \mathrm{g} / \mathrm{ml})$, served as stimulator cells. MLR was performed as reported. ${ }^{16}$ Cells were harvested after $72 \mathrm{~h}$ post culture and analysed for $\mathrm{T}$ cell subset surface markers (CD4, CD8, CD25, CD62L, CD44) and intracellular marker FOXP3 in the responder cells by flow 
cytometry; part of the cells was used for RNA extraction and cytokine gene expression analysis by RT-qPCR. Markers for phenotypes: central memory T cells (central Tm, CD44+CD62L+), effector memory T cells (effector Tm, CD44+), effector T cells (Teff, CD25+), regulatory T cells (Treg, FOXP3+).

Quantitative gene expression analysis. Total RNA extraction and qPCR was performed as described previously. ${ }^{16}$ List of qPCR primers can be found in the extended data table S3. Gene expression values were normalized with endogenous control GAPDH. Relative quantification of each target gene studied was calculated by the $\Delta \Delta$ CT method.

Bulk RNA Sequencing. Trafficked maternal and recipient cells were isolated from 1-week-old F1 hybrid neonates (groups consists of uninjected offspring (no IUT, DT-, $n=17$ ), mIUT (DT-, $n=7$ ), pIUT (DT-, $n=5)$ and DT+plUT $(n=5))$ by magnetic cell sorting (MACS), followed by FACS, using H2K-b and H2-Kd antibodies respectively. Isolated maternal trafficked cells from each neonate were not sufficient for RNA-seq analysis as individual samples. We pooled the samples from a group of neonates to generate sufficient number of cells for sorting and performed RNA-seq. Thus, we do not have replicates for RNAseq samples that were analysed, but each sample represents a certain number of harvested animals. We were unable to isolate sufficient samples from DT+ mIUT and alUT groups for analyses. Total RNA was isolated from the sorted cells using RNeasy micro kits (Qiagen) with on-column RNase-free DNase digestion and eluted carrier-free. Total RNA was quantified, and the quality assessed using Agilent 2100 Bioanalyzer (Agilent Technologies Inc., Santa Clara, CA), before cDNA library preparation. Samples were sequenced on a flow cell using HiSeq 4000 system (Illumina, San Diego, CA), with a read length of $150 \mathrm{bp}$ and 100 million reads per sample. The raw reads were analyzed with the RNAseq pipeline from nf-core ${ }^{60}$ using the reference genome (GRCm39) and gene annotation (M26) from GENCODE (https://www.gencodegenes.org/). The gene read count table generated by featureCount in the RNAseq pipeline was analysed using edgeR software. ${ }^{61,62}$ The dispersion value was set at 0.4 as recommended in edgeR for experimental setups with no replicates.

Gene expression. Using log of fold-change (logFC) data, we selected the most up- and down-regulated genes (1500 each, for a total of 3000) from each treatment group to organize using an online Venn diagram generator (http://bioinformatics.psb.ugent.be/webtools/Venn/) to single out common genes across all the groups. We analysed functions of enriched genes using the Database for Annotation, Visualisation and Integrated Discovery (DAVID v6.8, https://david.ncifcrf.gov/). ${ }^{63,64}$

TCR and BCR repertoire analyses. Raw reads were analyzed by MIXCR performed with settings of analysis of random fragments, RNA starting material and using the provided Mus musculus dataset, all others remaining 
as default settings. ${ }^{65,66}$ Results from MIXCR were imported into VDJtools (https://github.com/mikessh/vdjtools)

345 to plot the clonotypes in PlotQuantileStats. ${ }^{67}$

Detection of donor specific antibodies (DSA). Serum collected from harvested pups and serum collected after donor booster injections were used for DSA. For generating positive control sera, wild-type B6 mice were sensitized with maternal (B6/CD45.1), paternal $(B A L B / c)$ or allogenic $(C 3 H)$ splenocytes at a dose of $2 \mathrm{E}+7$ cells, injected intra-peritoneally at an interval of 2 weeks. Sera was collected after 14 days. DSA assay was performed using respective splenocytes, as described previously. ${ }^{16}$

351 Statistical analyses. Continuous data were analysed using Analysis of variance (2way ANOVA) with Tukey's 352 multiple comparisons test, with a single pooled variance and multiple t tests for comparisons of individual 353 parameters. Statistical significance was determined at $\alpha=5.0 \%$. Values are expressed as mean \pm standard error 354 of the mean (SEM). Pearson correlation coefficient was also used assuming linear relationships between the 355 variables tested. Analyses were performed with GraphPad Prism version 9 for Windows (GraphPad Software, 356 San Diego, CA, www.graphpad.com). Error bars were not provided on figure 2a-f, figure 3a-f and extended 357 figure S3a-f, as they may mask a clear view of the graph bars. Instead, respective raw data (mean \pm SEM), were 358 provided in the supplementary table 1-3). All experimental data were collected and analysis were performed by 359 separate team of staffs. Investigators were blinded for the current study. The datasets generated and/or 360 analysed during the current study are available from the corresponding author on reasonable request. 


\section{References}

3621 Mattar, C. N., Biswas, A., Choolani, M. \& Chan, J. K. The case for intrauterine stem cell transplantation. Best Pract Res Clin Obstet Gynaecol 26, 683-695, doi:10.1016/j.bpobgyn.2012.06.005 (2012). Troeger, C. et al. In utero haematopoietic stem cell transplantation. Experiences in mice, sheep and humans. Swiss Med Wkly. 137, 14S-19S (2007 Mar).

3 Merianos, D., Heaton, T. \& Flake, A. W. In utero hematopoietic stem cell transplantation: progress toward clinical application. Biol Blood Marrow Transplant 14, 729-740, doi:10.1016/j.bbmt.2008.02.012 (2008).

4 Shields, L. E. et al. Fetal immune suppression as adjunctive therapy for in utero hematopoietic stem cell transplantation in nonhuman primates. Stem Cells 22, 759-769, doi:10.1634/stemcells.22-5-759 (2004).

5 Dighe, N. M. et al. A comparison of intrauterine hemopoietic cell transplantation and lentiviral gene transfer for the correction of severe beta-thalassemia in a HbbTh3/+ murine model. Exp Hematol 62, 45-55, doi:10.1016/j.exphem.2018.03.006 (2018).

6 Cruz, F. M., Colbert, J. D., Merino, E., Kriegsman, B. A. \& Rock, K. L. The Biology and Underlying Mechanisms of Cross-Presentation of Exogenous Antigens on MHC-I Molecules. Annual Review of Immunology 35, 149-176, doi:10.1146/annurev-immunol-041015-055254 (2017).

7 Collin, M. \& Bigley, V. Human dendritic cell subsets: an update. Immunology 154, 3-20, doi:10.1111/imm.12888 (2018).

8 Guilliams, M. et al. Dendritic cells, monocytes and macrophages: a unified nomenclature based on ontogeny. Nat Rev Immunol 14, 571-578, doi:10.1038/nri3712 (2014).

9 Manicassamy, S. \& Pulendran, B. Dendritic cell control of tolerogenic responses. Immunological Reviews 241, 206-227, doi:https://doi.org/10.1111/j.1600-065X.2011.01015.x (2011).

10 Dudziak, D. et al. Differential Antigen Processing by Dendritic Cell Subsets in Vivo. Science 315, 107111, doi:doi:10.1126/science.1136080 (2007).

11 Moreau, A., Varey, E., Bouchet-Delbos, L. \& Cuturi, M.-C. Cell therapy using tolerogenic dendritic cells in transplantation. Transplantation Research 1, 13, doi:10.1186/2047-1440-1-13 (2012).

12 Silva, P. d. M. et al. Tolerogenic Dendritic Cells on Transplantation: Immunotherapy Based on Second Signal Blockage. J Immunol Res 2015, 856707-856707, doi:10.1155/2015/856707 (2015).

13 Sichien, D., Lambrecht, B. N., Guilliams, M. \& Scott, C. L. Development of conventional dendritic cells: from common bone marrow progenitors to multiple subsets in peripheral tissues. Mucosal Immunol 10, 831-844, doi:10.1038/mi.2017.8 (2017).

14 Jeanty, C., Derderian, S. C. \& Mackenzie, T. C. Maternal-fetal cellular trafficking: clinical implications and consequences. Curr Opin Pediatr 26, 377-382, doi:10.1097/MOP.0000000000000087 (2014).

15 Bianchi, D. Robert E. Gross Lecture. Fetomaternal cell trafficking: a story that begins with prenatal diagnosis and may end with stem cell therapy. J Pediatr Surg 42, 12-18, doi:S0022-3468(06)00646-4 [pii]10.1016/j.jpedsurg.2006.09.047 (2007).

16 Kandasamy, K. et al. Maternal microchimerism and cell-mediated immune-modulation enhance engraftment following semi-allogenic intrauterine transplantation. FASEB J 35, e21413, doi:10.1096/fj.202002185RR (2021).

17 Nijagal, A. et al. Maternal T cells limit engraftment after in utero hematopoietic cell transplantation in mice. J Clin Invest 121, 582-592, doi:10.1172/JCl44907 (2011).

18 Wegorzewska, M. et al. Fetal intervention increases maternal T cell awareness of the foreign conceptus and can lead to immune-mediated fetal demise. J Immunol 192, 1938-1945, doi:10.4049/jimmunol.1302403 (2014).

19 McGovern, N. et al. Human fetal dendritic cells promote prenatal T-cell immune suppression through arginase-2. Nature 546, 662-666, doi:10.1038/nature22795 (2017).

20 Mishra, A. et al. Microbial exposure during early human development primes fetal immune cells. Cell 184, 3394-3409.e3320, doi:10.1016/j.cell.2021.04.039 (2021).

21 Hochweller, K., Striegler, J., Hämmerling, G. J. \& Garbi, N. A novel CD11c.DTR transgenic mouse for depletion of dendritic cells reveals their requirement for homeostatic proliferation of natural killer cells. Eur J Immunol 38, 2776-2783, doi:10.1002/eji.200838659 (2008). 
Abdoli, R. \& Najafian, N. T Helper Cells Fate Mapping by Co-stimulatory Molecules and its Functions in Allograft Rejection and Tolerance. Int J Organ Transplant Med 5, 97-110 (2014).

23 Noh, J., Choi, W. S., Noh, G. \& Lee, J. H. Presence of Foxp3-expressing CD19(+)CD5(+) B Cells in Human Peripheral Blood Mononuclear Cells: Human CD19(+)CD5(+)Foxp3(+) Regulatory B Cell (Breg). Immune Netw 10, 247-249, doi:10.4110/in.2010.10.6.247 (2010).

24 Tian, J. et al. Lipopolysaccharide-activated B cells down-regulate Th1 immunity and prevent autoimmune diabetes in nonobese diabetic mice. J Immunol 167, 1081-1089, doi:10.4049/jimmunol.167.2.1081 (2001).

25 Alberdi, A. \& Gilbert, M. T. P. A guide to the application of Hill numbers to DNA-based diversity analyses. Mol Ecol Resour 19, 804-817, doi:10.1111/1755-0998.13014 (2019).

26 Laydon, D. J., Bangham, C. R. M. \& Asquith, B. Estimating T-cell repertoire diversity: limitations of classical estimators and a new approach. Philos Trans R Soc Lond B Biol Sci 370, 20140291, doi:10.1098/rstb.2014.0291 (2015).

27 Hou, X. et al. Shorter TCR $\beta$-Chains Are Highly Enriched During Thymic Selection and Antigen-Driven Selection. Front Immunol 10, 299, doi:10.3389/fimmu.2019.00299 (2019).

28 Gupta, S., Stamatoyannopoulos, J. A., Bailey, T. L. \& Noble, W. S. Quantifying similarity between motifs. Genome Biol 8, R24, doi:10.1186/gb-2007-8-2-r24 (2007).

29 Waaga, A. M. et al. Regulatory functions of self-restricted MHC class II allopeptide-specific Th2 clones in vivo. J Clin Invest 107, 909-916, doi:10.1172/jci11427 (2001).

30 Kim, H. B., Shaaban, A. F., Yang, E. Y., Liechty, K. W. \& Flake, A. W. Microchimerism and tolerance after in utero bone marrow transplantation in mice. The Journal of surgical research 77, 1-5, doi:10.1006/jsre.1997.5255 (1998).

31 Hayashi, S., Peranteau, W. H., Shaaban, A. F. \& Flake, A. W. Complete allogeneic hematopoietic chimerism achieved by a combined strategy of in utero hematopoietic stem cell transplantation and postnatal donor lymphocyte infusion. Blood 100, 804-812, doi:10.1182/blood-2002-01-0016 (2002).

32 Taylor, P. A., McElmurry, R. T., Lees, C. J., Harrison, D. E. \& Blazar, B. R. Allogenic fetal liver cells have a distinct competitive engraftment advantage over adult bone marrow cells when infused into fetal as compared with adult severe combined immunodeficient recipients. Blood 99, 1870-1872, doi:10.1182/blood.V99.5.1870 (2002).

33 Schoeberlein, A., Holzgreve, W., Dudler, L., Hahn, S. \& Surbek, D. V. In utero transplantation of autologous and allogeneic fetal liver stem cells in ovine fetuses. American journal of obstetrics and gynecology 191, 1030-1036, doi:10.1016/j.ajog.2004.06.042 (2004).

34 Peranteau, W. H. et al. CD26 inhibition enhances allogeneic donor-cell homing and engraftment after in utero hematopoietic-cell transplantation. Blood 108, 4268-4274, doi:10.1182/blood-2006-04018986 (2006).

35 Kim, A. G. et al. Enhanced in utero allogeneic engraftment in mice after mobilizing fetal HSCs by alpha4beta1/7 inhibition. Blood, doi:10.1182/blood-2016-06-723981 (2016).

36 Vrecenak, J. D. et al. Preclinical Canine Model of Graft-versus-Host Disease after In Utero Hematopoietic Cell Transplantation. Biology of blood and marrow transplantation : journal of the American Society for Blood and Marrow Transplantation 24, 1795-1801, doi:10.1016/j.bbmt.2018.05.020 (2018).

$37 \mathrm{Fu}, \mathrm{S}$. et al. CD4+ CD25+ CD62+ T-regulatory cell subset has optimal suppressive and proliferative potential. Am J Transplant 4, 65-78, doi:10.1046/j.1600-6143.2003.00293.x (2004).

38 Ermann, J. et al. Only the CD62L+ subpopulation of CD4+CD25+ regulatory T cells protects from lethal acute GVHD. Blood 105, 2220-2226, doi:10.1182/blood-2004-05-2044 (2005).

39 Ghendler, Y. et al. Differential thymic selection outcomes stimulated by focal structural alteration in peptide/major histocompatibility complex ligands. Proceedings of the National Academy of Sciences 95, 10061-10066, doi:10.1073/pnas.95.17.10061 (1998).

40 Rocha, B. \& Boehmer, H. v. Peripheral Selection of the T Cell Repertoire. Science 251, 1225-1228, doi:doi:10.1126/science.1900951 (1991).

$41 \mathrm{Wu}, \mathrm{C}$. J. et al. Reconstitution of T-cell receptor repertoire diversity following T-cell depleted allogeneic bone marrow transplantation is related to hematopoietic chimerism. Blood 95, 352-359 (2000). 
Vernochet, C., Caucheteux, S. M. \& Kanellopoulos-Langevin, C. Bi-directional Cell Trafficking Between Mother and Fetus in Mouse Placenta. Placenta 28, 639-649, doi:https://doi.org/10.1016/j.placenta.2006.10.006 (2007).

$43 \mathrm{Li}, \mathrm{Y}$. \& $\mathrm{Xu}, \mathrm{L}$. Evaluation of TCR repertoire diversity in patients after hematopoietic stem cell transplantation. Stem Cell Investig 2, 17-17, doi:10.3978/j.issn.2306-9759.2015.09.01 (2015).

44 Muraro, P. A. et al. T cell repertoire following autologous stem cell transplantation for multiple sclerosis. The Journal of Clinical Investigation 124, 1168-1172, doi:10.1172/JCI71691 (2014).

45 van Heijst, J. W. et al. Quantitative assessment of T cell repertoire recovery after hematopoietic stem cell transplantation. Nat Med 19, 372-377, doi:10.1038/nm.3100 (2013).

46 Bizargity, P. \& Bonney, E. A. Dendritic cells: a family portrait at mid-gestation. Immunology 126, 565578, doi:10.1111/j.1365-2567.2008.02918.x (2009).

47 Erlebacher, A., Vencato, D., Price, K. A., Zhang, D. \& Glimcher, L. H. Constraints in antigen presentation severely restrict $T$ cell recognition of the allogeneic fetus. J Clin Invest 117, 1399-1411, doi:10.1172/jci28214 (2007).

48 Bonney, E. A. \& Matzinger, P. The maternal immune system's interaction with circulating fetal cells. J Immunol 158, 40-47 (1997).

49 Perchellet, A. L., Jasti, S. \& Petroff, M. G. Maternal CD4 ${ }^{+}$and CD8 ${ }^{+}$T cell tolerance towards a fetal minor histocompatibility antigen in $\mathrm{T}$ cell receptor transgenic mice. Biol Reprod 89, 102, doi:10.1095/biolreprod.113.110445 (2013).

50 Tafuri, A., Alferink, J., Möller, P., Hämmerling, G. J. \& Arnold, B. T cell awareness of paternal alloantigens during pregnancy. Science 270, 630-633, doi:10.1126/science.270.5236.630 (1995).

51 Aït-Azzouzene, D. et al. Maternal B lymphocytes specific for paternal histocompatibility antigens are partially deleted during pregnancy. J Immunol 161, 2677-2683 (1998).

52 Gupta, P. K., Mclntosh, C. M., Chong, A. S. \& Alegre, M.-L. The pursuit of transplantation tolerance: new mechanistic insights. Cell Mol Immunol 16, 324-333, doi:10.1038/s41423-019-0203-7 (2019).

53 Graca, L. et al. Both $\mathrm{CD} 4(+) \mathrm{CD} 25(+)$ and $\mathrm{CD} 4(+) \mathrm{CD} 25(-)$ regulatory cells mediate dominant transplantation tolerance. J Immunol 168, 5558-5565, doi:10.4049/jimmunol.168.11.5558 (2002).

54 Francis, R. S. et al. Induction of transplantation tolerance converts potential effector T cells into graftprotective regulatory T cells. European journal of immunology 41, 726-738, doi:10.1002/eji.201040509 (2011).

55 Young, J. S. et al. Erosion of Transplantation Tolerance After Infection. American journal of transplantation : official journal of the American Society of Transplantation and the American Society of Transplant Surgeons 17, 81-90, doi:10.1111/ajt.13910 (2017).

56 Delgoffe, G. M. et al. Stability and function of regulatory T cells is maintained by a neuropilin-1semaphorin-4a axis. Nature 501, 252-256, doi:10.1038/nature12428 (2013).

57 Wang, L., Fu, Y. \& Chu, Y. in B Cells in Immunity and Tolerance (ed Ji-Yang Wang) 87-103 (Springer Singapore, 2020).

58 Klinker, M. W. \& Lundy, S. K. Multiple mechanisms of immune suppression by B lymphocytes. Mol Med 18, 123-137, doi:10.2119/molmed.2011.00333 (2012).

59 Achilli, C., Duran-Retamal, M., Saab, W., Serhal, P. \& Seshadri, S. The role of immunotherapy in in vitro fertilization and recurrent pregnancy loss: a systematic review and meta-analysis. Fertil Steril 110, 1089-1100, doi:10.1016/j.fertnstert.2018.07.004 (2018).

60 nf-core/rnaseq: nf-core/rnaseq version 1.4.2 (1.4.2). v. 1.4.2 (Zenodo, 2019).

61 McCarthy, D. J., Chen, Y. \& Smyth, G. K. Differential expression analysis of multifactor RNA-Seq experiments with respect to biological variation. Nucleic Acids Res 40, 4288-4297, doi:10.1093/nar/gks042 (2012).

62 Robinson, M. D., McCarthy, D. J. \& Smyth, G. K. edgeR: a Bioconductor package for differential expression analysis of digital gene expression data. Bioinformatics 26, 139-140, doi:10.1093/bioinformatics/btp616 (2010).

63 Huang da, W., Sherman, B. T. \& Lempicki, R. A. Bioinformatics enrichment tools: paths toward the comprehensive functional analysis of large gene lists. Nucleic Acids Res 37, 1-13, doi:10.1093/nar/gkn923 (2009).

64 Huang da, W., Sherman, B. T. \& Lempicki, R. A. Systematic and integrative analysis of large gene lists using DAVID bioinformatics resources. Nat Protoc 4, 44-57, doi:10.1038/nprot.2008.211 (2009). 
51965 Bolotin, D. A. et al. Antigen receptor repertoire profiling from RNA-seq data. Nat Biotechnol 35, 908380-381, doi:10.1038/nmeth.3364 (2015).

67 Shugay, M. et al. VDJtools: Unifying Post-analysis of T Cell Receptor Repertoires. PLoS Comput Biol 11, e1004503, doi:10.1371/journal.pcbi.1004503 (2015). 
Table 1: RNAseq analysis of trafficked maternal and recipient cells for TCR and BCR repertoire 530

\begin{tabular}{|c|c|c|c|c|}
\hline Sample & $\begin{array}{l}\text { Total } \\
\text { counts }\end{array}$ & $\begin{array}{l}\text { Clonotype } \\
\text { number }\end{array}$ & $\begin{array}{l}\text { Mean } \\
\text { clonotype } \\
\text { frequency }\end{array}$ & $\begin{array}{l}\text { Hill numbers } \\
\text { (order 1) } \\
\text { *exponential of the } \\
\text { Shannon-Weiner } \\
\text { Index }\end{array}$ \\
\hline \multicolumn{5}{|c|}{ Trafficked maternal immune cells } \\
\hline Uninjected pup & 348684 & 4429 & $2.26 \mathrm{E}-4$ & 941 \\
\hline mIUT recipient & 8376 & 49 & $2.04 \mathrm{E}-2$ & 29 \\
\hline plUT recipient & 3749 & 32 & $3.13 E-2$ & 18 \\
\hline $\begin{array}{l}\text { DT+ plUT recipient } \\
\text { (maternal DC suppression) }\end{array}$ & 5537 & 552 & $1.81 \mathrm{E}-3$ & 447 \\
\hline \multicolumn{5}{|l|}{ Fetal immune cells } \\
\hline Uninjected pup & 66848 & 148 & $6.76 \mathrm{E}-4$ & 64 \\
\hline mIUT recipient & 4043 & 666 & $1.50 \mathrm{E}-3$ & 528 \\
\hline plUT recipient & 48175 & 5488 & $1.82 \mathrm{E}-4$ & 3728 \\
\hline $\begin{array}{l}\text { DT+ plUT recipient } \\
\text { (maternal DC suppression) }\end{array}$ & 18579 & 3064 & $3.26 \mathrm{E}-4$ & 1637 \\
\hline
\end{tabular}



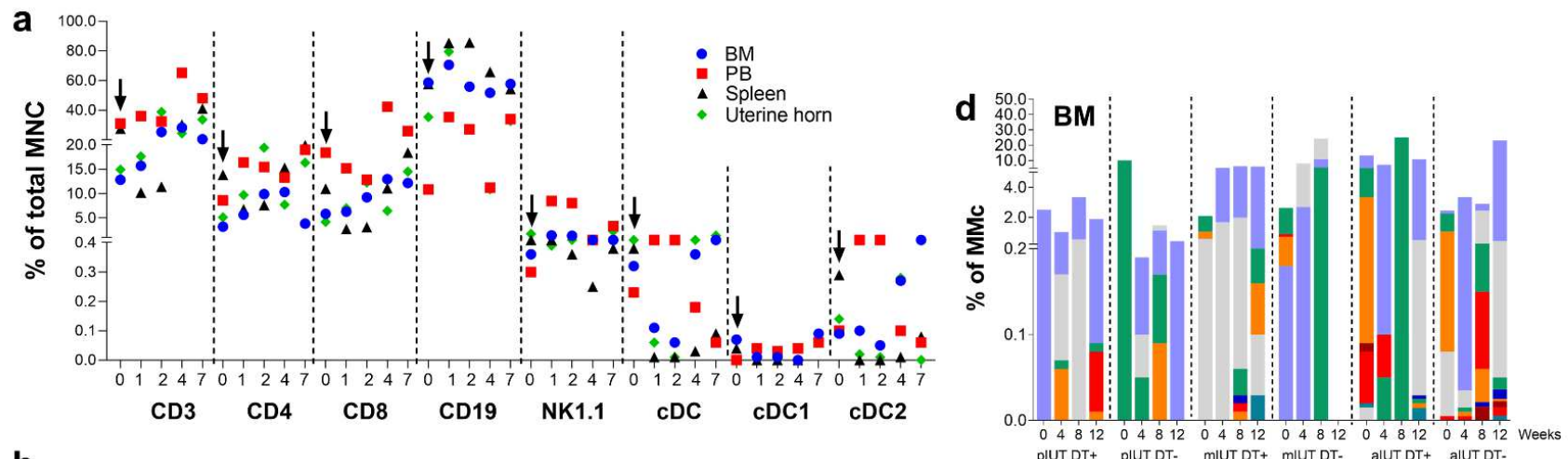

b
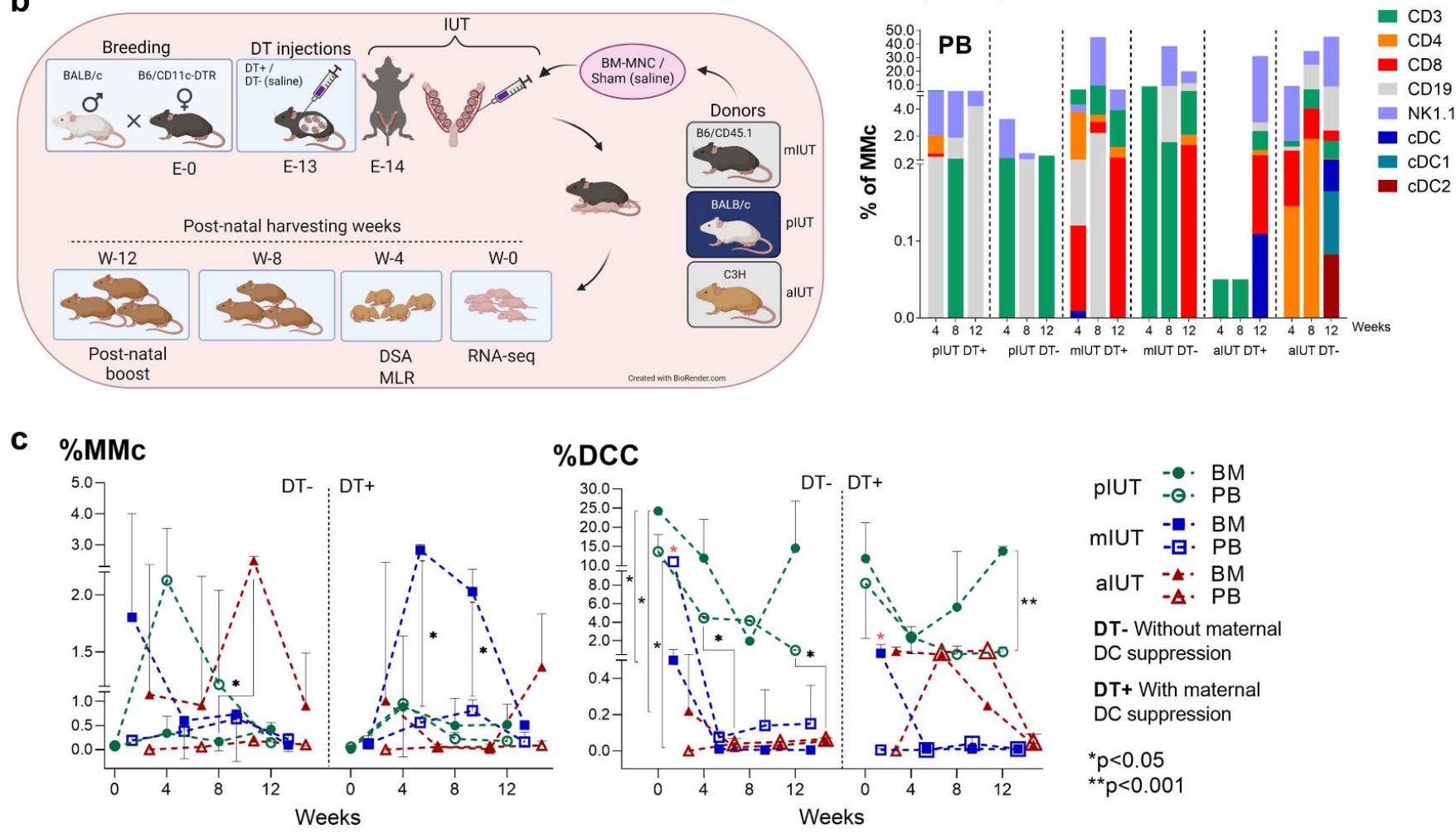

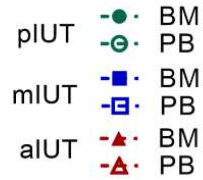

DT- Without maternal DC suppression

DT+ With maternal

DC suppression

${ }^{*} p<0.05$

Fig. 1: Transient suppression of maternal dendritic cells with diphtheria toxin resulted in reduced maternal immune cell trafficking to fetal recipients following intrauterine transplantation of semiallogenic and fully allogenic donor cells, and effect on donor cell chimerism

Selective reduction of conventional dendritic cell (cDC) and subtypes (cDC1, cDC2) in various organs of nonpregnant CD11c.DTR females $(n=5)$ after administering diphtheria toxin (DT, arrows, a). Intrauterine transplantations of maternal $(n=31$, mIUT) paternal $(n=60$, plUT) and fully allogenic $(n=22$, alUT) cells performed at E14 (b), with (DT+) or without DT (DT-). Maternal immune cell microchimerism (MMc) was similar in DT- and DT + with each IUT (c), higher in alUT $\vee$ plUT, and higher in DT+ bone marrow (BM) $v$ peripheral blood (PB) after mIUT (black*). Donor cell chimerism (DCC) was highest with DT- plUT in BM and PB (black*), and higher in mIUT PB v BM (red*). With DT+, DCC was maintained in plUT BM (black*), and was higher in mIUT BM v PB (red*). Maternal cDC, CD3, CD4, CD19 cells were non-significantly reduced in DT+ recipient BM and higher in PB (d). CDC and subtypes were only found in DT-and DT+ alUT recipients. Data represent mean+SEM, analysed by two-way ANOVA with Tukey's multiple comparisons test. 

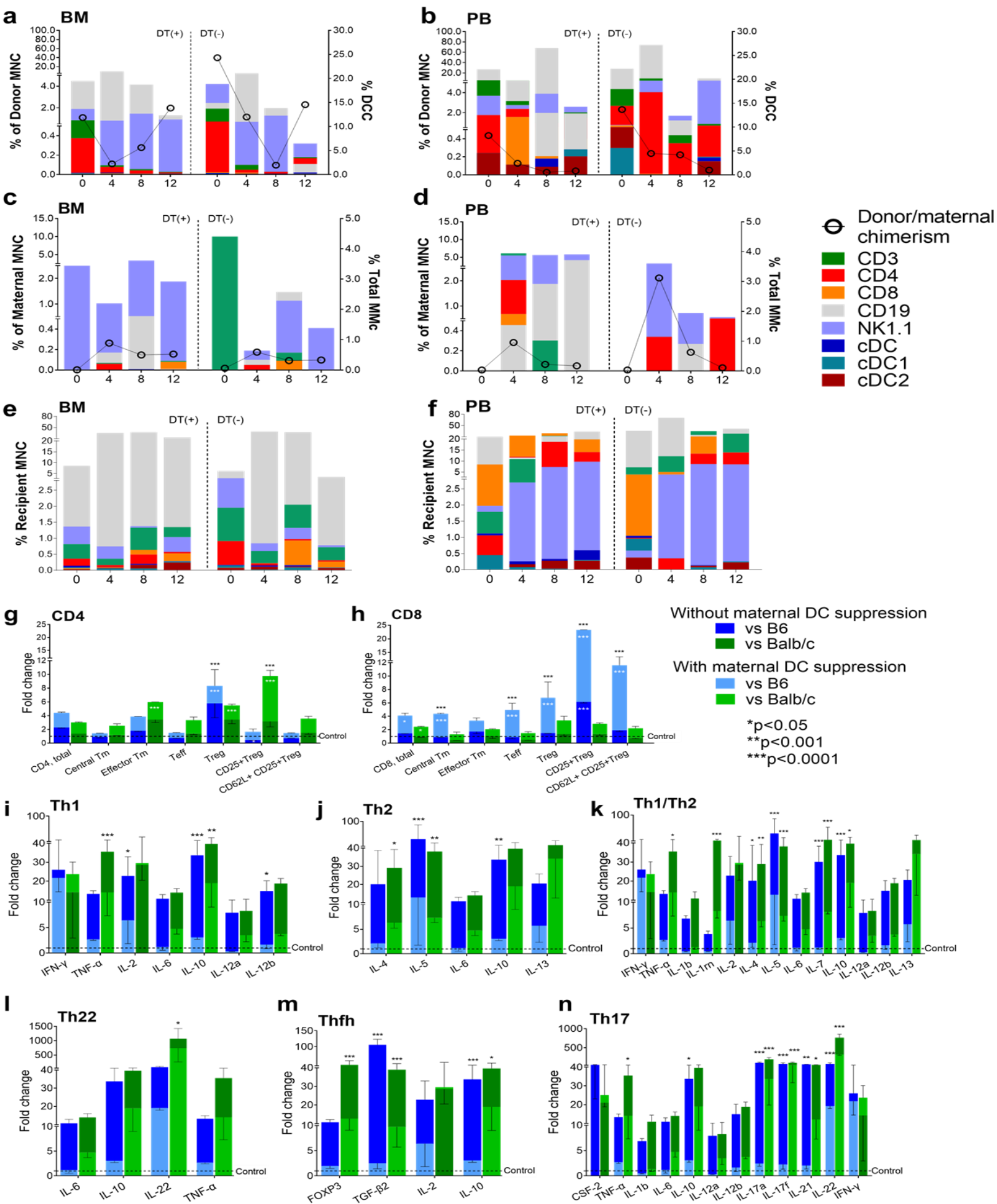

Fig. 2: Immune profiles of engrafted donor, trafficked maternal and recipient cells, $T$ cell profile and cytokine expression following paternal cell transplantation

Compared to DT- $(n=9), D T+$ recipients $(n=11)$ had engrafted donor cells showing reductions in all immune types relative to $\operatorname{CD} 19(p<0.05, \mathbf{a}, \mathbf{b})$, and maternal microchimerism with lower levels of all immune cells, and lower cDC, CD19, NK1.1 relative to CD3 ( $<<0.05, \mathbf{c}, \mathbf{d})$, in bone marrow (BM) and peripheral blood (PB) respectively. Recipient immune cell profile was similar in DT+ and DT-, with relatively higher CD19 in both $(p<0.005$, e,f). Weeks shown on $x$-axis. DT+ $(n=4)$ showed higher fold-changes in CD4+ and CD8+ $(\mathbf{g}, \mathbf{h})$ central and effector memory and regulatory T cells compared to uninjected controls $\left(n=3\right.$, white $\left.{ }^{*}\right)$ and to DT- $(n=3$, black*). Reductions in helper T cell cytokines (i-n) were observed with $D T+(n=4)$ in response to paternal and maternal donor cells, compared to DT- $\left(n=4\right.$, black $\left.{ }^{*}\right)$. Data represent mean \pm SEM, analysed by two-way ANOVA with 

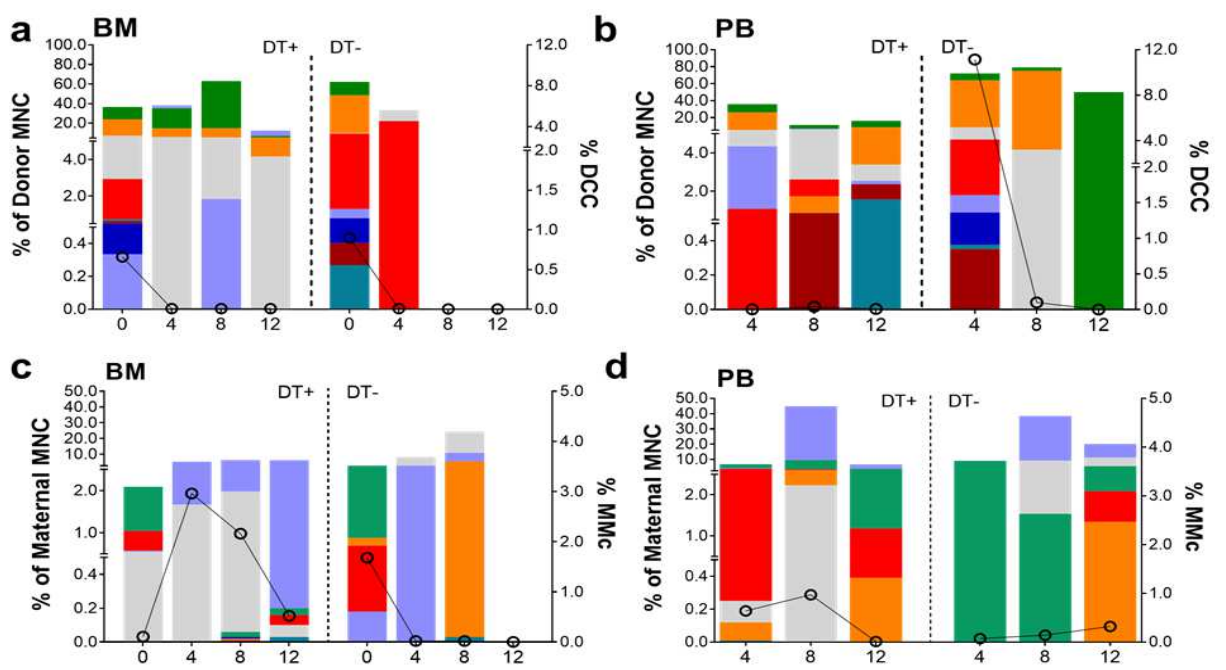

d

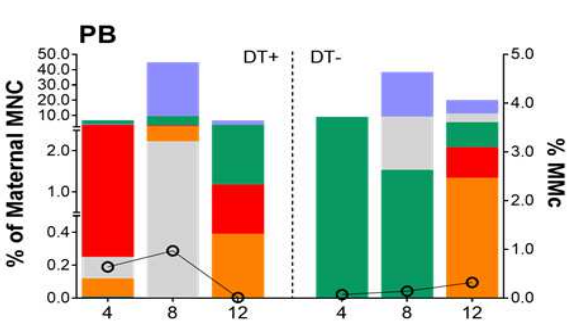

Donor/maternal chimerism - CD3 - CD4 CD19 - NK1.1 cDC CDC2

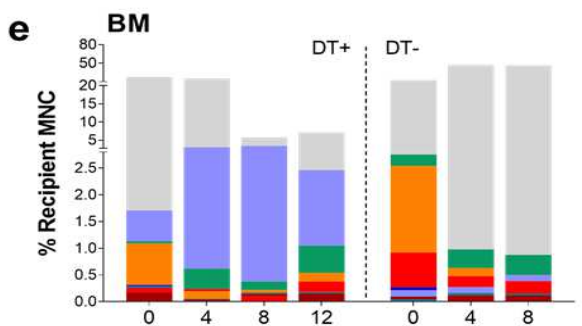

f
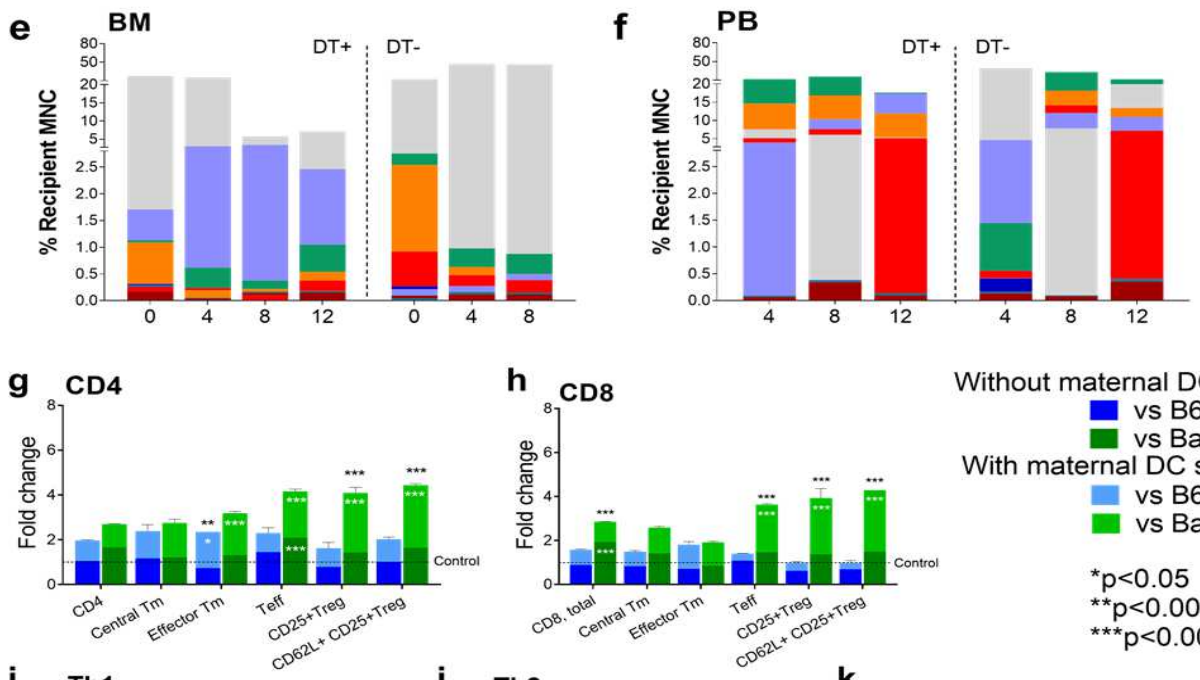

Without maternal DC suppression
vs B6

$$
\begin{aligned}
& \text { vs B6 } \\
& \text { vs Balb/c }
\end{aligned}
$$

With maternal DC suppression

$$
\text { vs B6 }
$$

- vs Balb/c

${ }^{*} \mathrm{p}<0.05$

${ }^{* *} p<0.001$

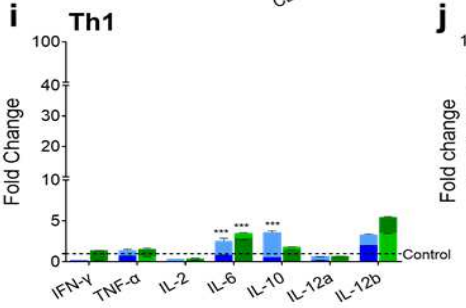

$\mathrm{j}_{100}{ }^{\mathrm{Th} 2}$

k Th1/Th2

${ }^{\star * \star} p<0.0001$
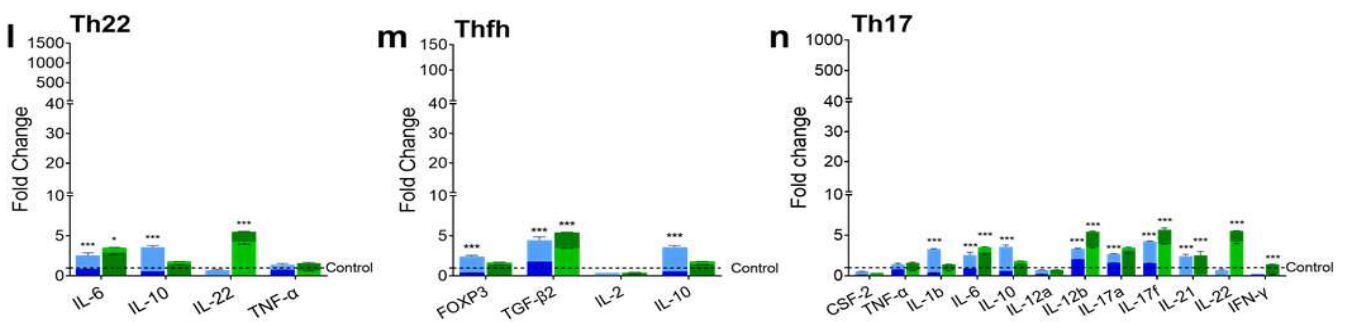

Fig 3: Immune profiles of engrafted donor, trafficked maternal and recipient cells, $T$ cell profile and cytokine expression following maternal cell transplantation

Donor cell chimerism was low in DT+ $(n=8)$ and DT- $(n=7)$. In DT+, CD3 was higher in BM and lower in PB $(\mathbf{p}<0.05)$ than in DT- $(\mathbf{a}, \mathbf{b})$. MMc $(\mathbf{c}, \mathbf{d})$ and recipient immune cell profiles $(\mathbf{e}, \mathbf{f})$ were similar in DT+ and DT-. Weeks shown on x-axis. CD4+ effector memory, CD4+ and CD8+ effector and regulatory T cells in DT+ $(n=4)$ were increased over DT- $(n=3$, black $)$ and over uninjected controls $(n=3 \text {, white })^{*}$ following paternal donor cell exposure $(\mathbf{g}, \mathbf{h})$. DT+ $(n=4)$ and DT- $(n=4)$ produced similar low-level responses in individual cytokines over untreated controls $\left(n=2\right.$, black $\left.{ }^{*}\right)$ which were much lower than in paternal IUT ( $y$-axis scale same as Figure 2i-n). DT+ produced higher expression of largely inhibitory cytokines associated with Th2, Th1/Th2, Th17 cells, following stimulation (i-n). Data represent mean \pm SEM, analysed by two-way ANOVA with Tukey's multiple comparisons test. 

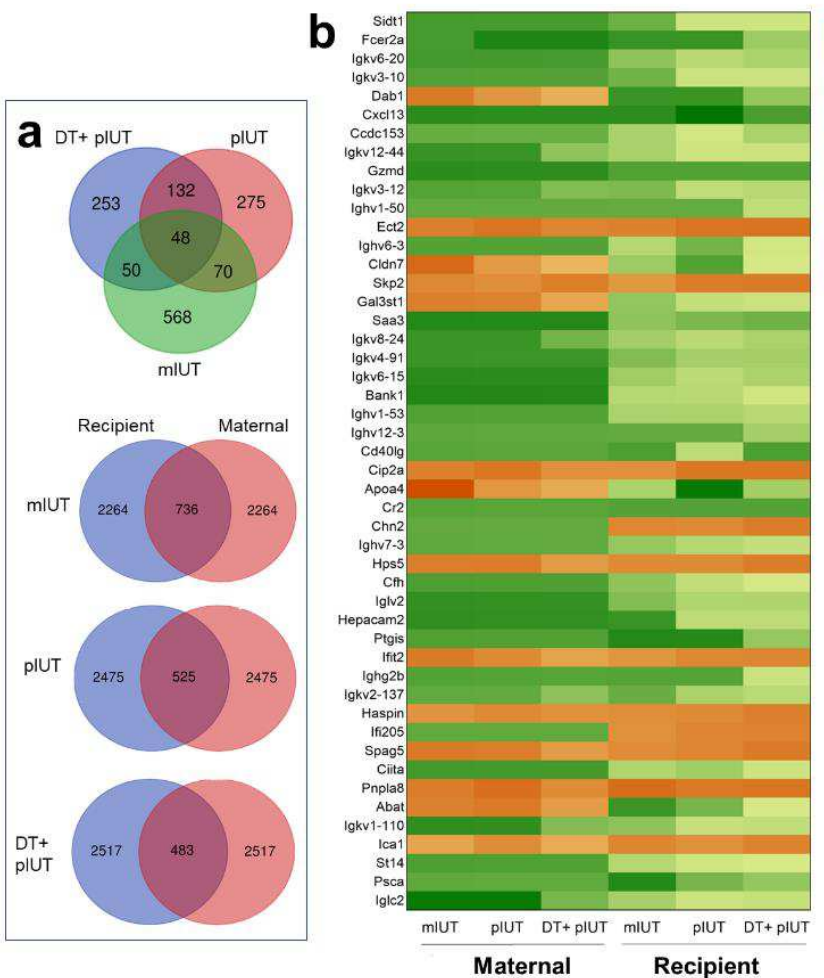

d Trafficked maternal immune cell clonotypes Uninjected recipient mIUT recipient plUT recipient DT+ pIUT recipient

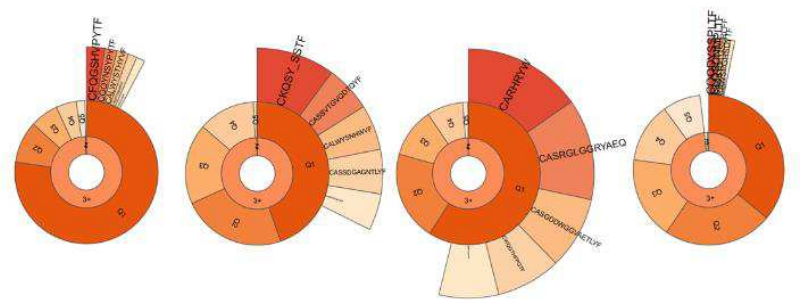

Fetal immune cell clonotypes

e

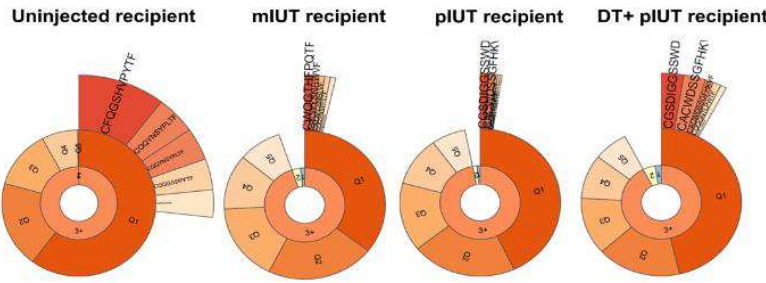

C

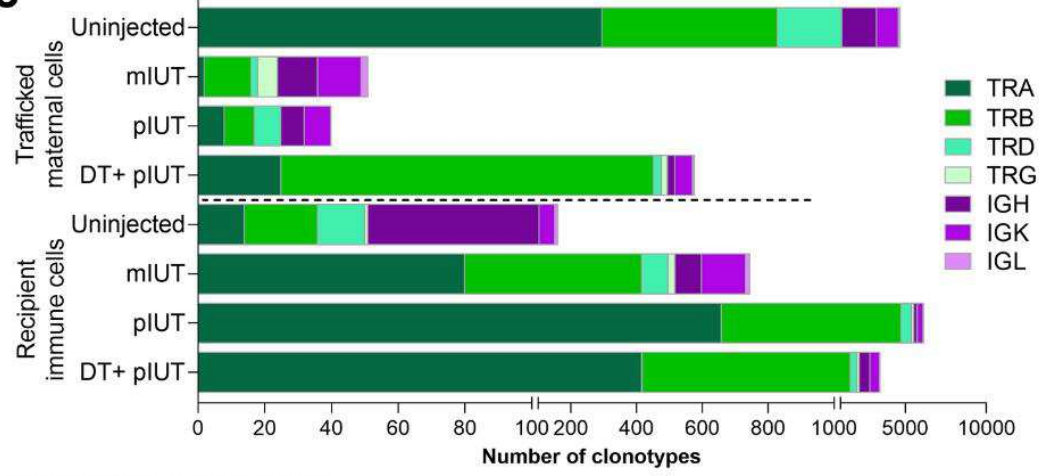

f Maternal immune cells

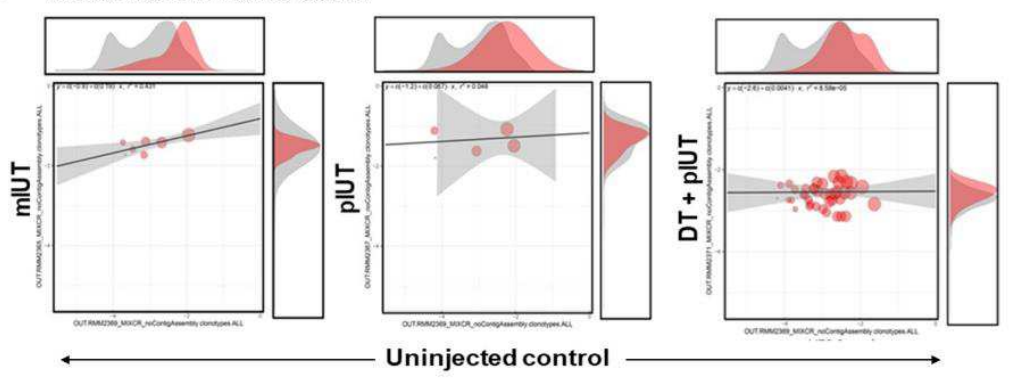

g Recipient immune cells

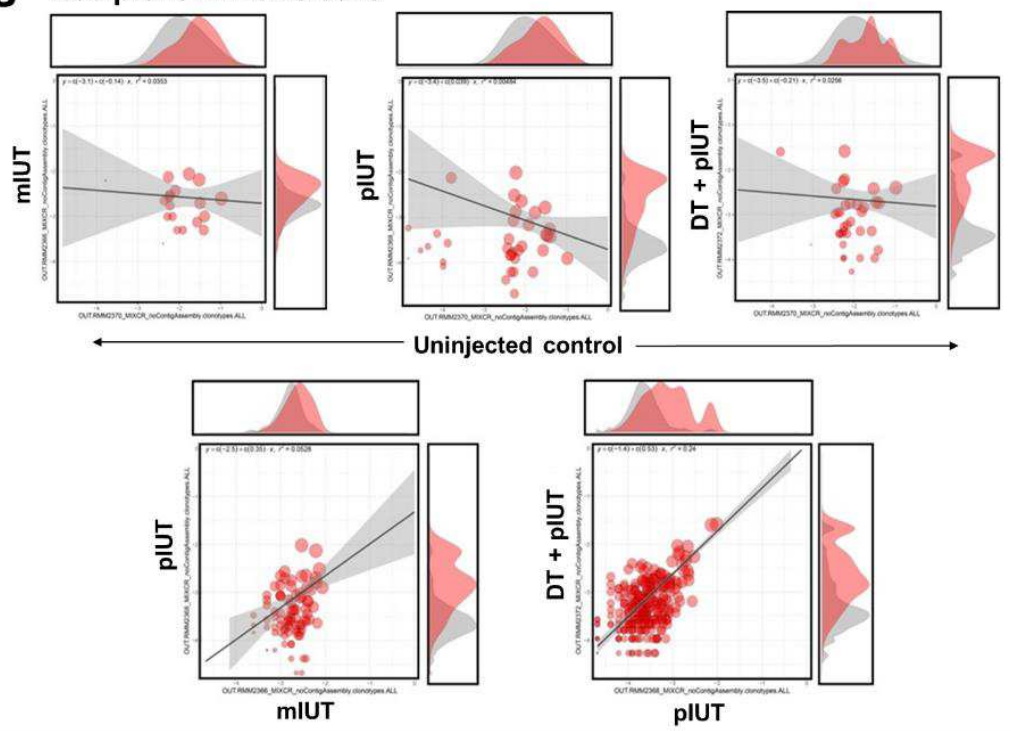

Fig. 4: Gene expression and T cell (TCR) and B cell (BCR) receptor repertoire of trafficked maternal and recipient immune cells.

Maternal and recipient immune cells from pIUT (DT $+n=5$, DT- $n=5)$, DT-mIUT $(n=7)$ shared 48 genes (top panel) and up to $30 \%$ genes (bottom panel) within each IUT (a). Common gene clusters represent cell adhesion, cytokine response and immunoregulatory pathways (b). Maternal- and recipient-derived BCR and TCR clonotypes (c) were similar in uninjected pups $(n=17)$; IUT increased TRB, TRG, reduced TRA, TRD, IGH, IGK in maternal-derived and recipient-derived clonotypes. Top 5 maternal-derived clonotypes were greatest following IUT and contracted in DT+pIUT (d). Recipient-derived top 5 clonotypes were most abundant in uninjected pups (e). Large overlaps observed between DT+plUT and uninjected maternal-derived clonotypes (f) and between IUT recipients $(\mathbf{g})$. 


\section{Supplementary Files}

This is a list of supplementary files associated with this preprint. Click to download.

- SupplementaryInformation181221.pdf

- ExtendeddataNATURE181221.pdf

- rs.pdf 